\title{
Persistent headache after earache
}

\section{Anita Au MD, Mahdiya Al Bulushi MD, Mashel Al Rajub MD, Suzanne Morin MD MSc}

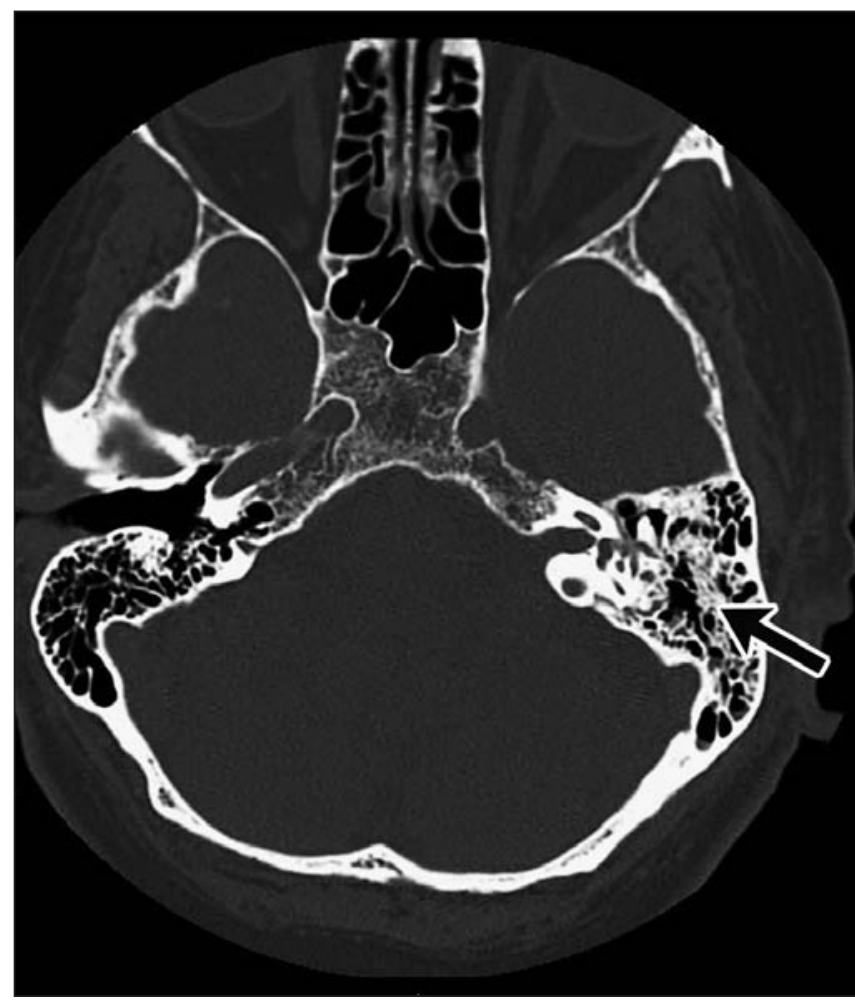

Figure 1: Computed tomogram showing fluid in the mastoid air cells on the left side (arrow) consistent with mastoiditis in a 67-year-old woman who presented with headache, neck pain, nausea and vomiting.

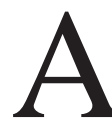

67-year-old woman with a history of diabetes, hypertension and remote deep vein thrombosis in her calf following colon surgery presented to her family physician with a seven-day history of pain in her left ear, nausea, vomiting and vertigo. Otitis media was diagnosed and was treated with oral antibiotics.

Two weeks later, the woman presented to the hospital following the onset of headache, neck pain, nausea and vomiting. She was afebrile. Examination of her head and neck revealed tenderness over the left mastoid process. The results of a neurologic examination were normal. Mastoiditis was confirmed by a non-infused computed tomography (CT) of

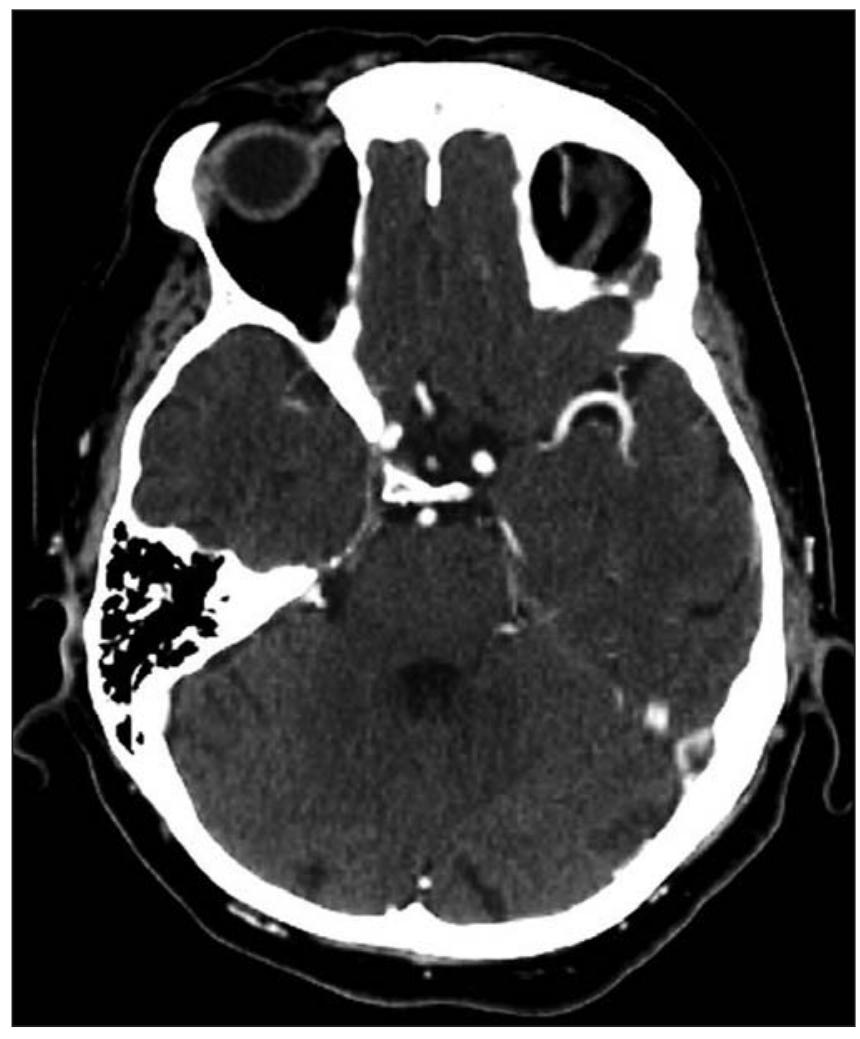

Figure 2: Contrast-enhanced computed tomogram of the head.

the head (Figure 1), and high-dose intravenous antibiotic treatment was prescribed. The following day, the patient had a tonic-clonic seizure. Examination of the cerebral spinal fluid excluded meningitis, and the result of polymerase chain reaction testing for herpesvirus was negative. She continued to have persistent headache and left-sided facial and aural pain. A contrast-enhanced CT scan of the head was ordered (Figure 2).

From the Department of Medicine (Au), Jewish General Hospital, McGil University, Montréal, Que.; and the Departments of Medicine and Radiology (Al Bulushi, Al Rajub, Morin), McGill University Health Centre, McGill University, Montréal, Que.

CMAJ 2011. DOI:10.1503/cmaj.091070 


\section{What is your diagnosis?}
a. Brain abscess
b. Ischemic stroke
c. Intracerebral hemorrhage
d. Cerebral venous sinus thrombosis
e. Cavernous sinus thrombosis
f. Giant cell arteritis

Based on the clinical presentation of a persistent headache and the radiologic evidence in the CT scans (Figure 3), our working diagnosis was (d) cerebral venous sinus thrombosis secondary to mastoiditis. Results of a work-up for a major thrombophilia defect were negative. Subsequent magnetic resonance imaging (MRI) and magnetic resonance venography (Figures 4 and 5) confirmed the diagnosis of cerebral venous sinus thrombosis. Heparin was given intravenously, and the patient subsequently received a vitamin $\mathrm{K}$ antagonist for three months. The headache gradually resolved with no neurologic sequelae.

\section{Discussion}

Cerebral venous sinus thrombosis is relatively rare, with an estimated annual incidence of three to four per million; $75 \%$ of those affected are women. ${ }^{1}$ Risk factors include acquired and genetic thrombophilia defects; pregnancy; infections such as otitis media, mastoiditis and meningitis; inflammatory diseases; malignant disease; drugs, such as oral contraceptives;

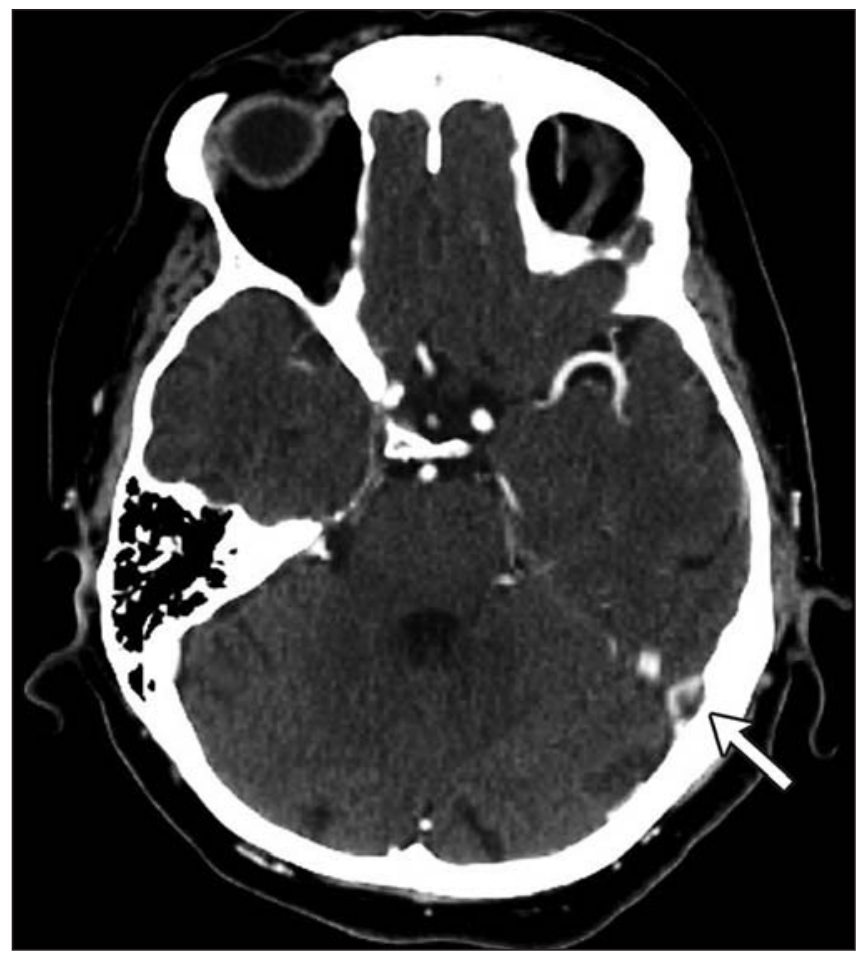

Figure 3: Contrast-enhanced computed tomogram showing a filling defect (thrombus) outlined by contrast medium in the left sigmoid sinus (arrow). This "empty triangle" or delta sign appearance of the confluens sinuum is frequently observed with cerebral venous sinus thrombosis. head injury; and lumbar puncture. ${ }^{1}$ A direct cause of thrombophilia is found in $85 \%$ of patients. ${ }^{1}$ Infection was present in $12.3 \%$ of patients in a multinational prospective observational study involving adults with sinus thrombosis. ${ }^{2}$

The clinical manifestation of cerebral venous sinus thrombosis is highly variable. The average delay is seven days from the onset of symptoms to diagnosis. ${ }^{2}$ The condition should be considered in patients with persistent headache or stroke-like symptoms in the absence of vascular risk factors. Box 1 outlines some of the differential diagnoses we considered for our patient. ${ }^{1-6}$ Headache, which occurs in $90 \%$ of patients, ${ }^{1}$ is frequently severe and diffuse in nature and often precedes the onset of neurologic symptoms. ${ }^{5}$ Behavioural symptoms, such as delirium, amnesia and mutism, can occur if the deep venous system is involved. ${ }^{1}$ Twenty to forty per cent of patients may present with isolated intracranial hypertension and symptoms of headache, vomiting and blurred vision due to papilledema, in the absence of localizing neurologic findings. ${ }^{6}$ Elderly patients tend to have a distinctive clinical presentation with depressed consciousness or changes in mental status as opposed to isolated intracranial hypertension.?

Focal or generalized seizure, central motor deficits, sensory deficits, aphasia or hemianopia develops in $40 \%-60 \%$ of patients. ${ }^{6}$ A multicentre prospective observational study involving more than 600 patients reported that $39.3 \%$ had seizures at presentation. ${ }^{8}$ Alternating focal deficits are rare but characteristic of cerebral venous sinus thrombosis. ${ }^{1}$ Funduscopy will reveal papilledema if intracranial hypertension is

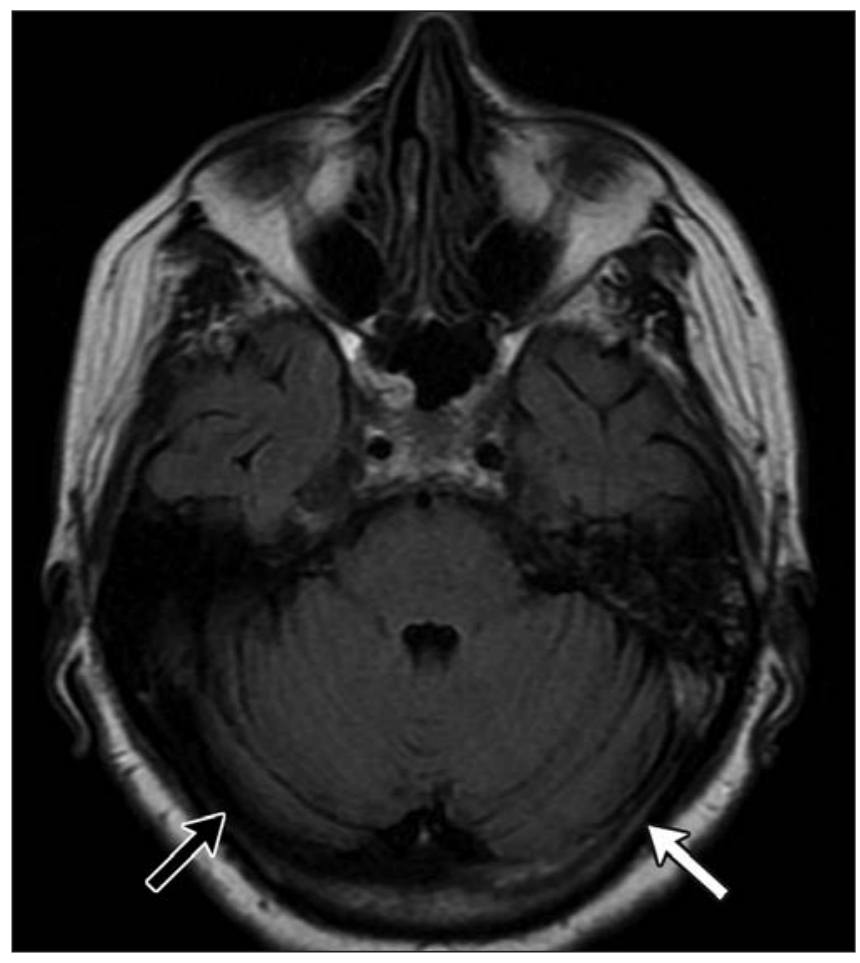

Figure 4: Axial FLAIR (fluid attenuated inversion recovery) magnetic resonance image showing abnormal bright signal in the left transverse sinus consistent with a thrombus (white arrow), compared with the absence of signal in the normal right transverse sinus (black arrow). 
present. The presence of hemorrhagic infarcts on CT scans, especially if there is more than one infarct, should also prompt the clinician to consider cerebral venous sinus thrombosis.

\section{Diagnosis}

Cerebral venous sinus thrombosis is diagnosed by means of neuroimaging. The best approach is the combination of MRI showing an abnormal signal in a sinus (Figure 4) in conjunction with magnetic resonance venography showing a corresponding absence of blood flow (Figure 5). ${ }^{1,6,9,10}$ During the first few days, the thrombus appears isointense on $T_{1}$ weighted images and hypointense on $T_{2}$-weighted images. Magnetic resonance venography is needed to rule out patency and show the absence of blood flow. The signal then becomes hyperintense, first on $T_{1}$-weighted then on $T_{2}$ weighted spin echo images. After one month, the signal becomes isointense on $T_{1}$-weighted images, with an increased signal on $T_{2}$-weighted images. ${ }^{59}$ The use of echoplanar susceptibility-weighted $T_{2}$ imaging may be particularly useful during the first three days of acute cerebral venous sinus thrombosis, when $T_{1^{-}}$and $T_{2}$-weighted images lack sensitivity. ${ }^{10}$

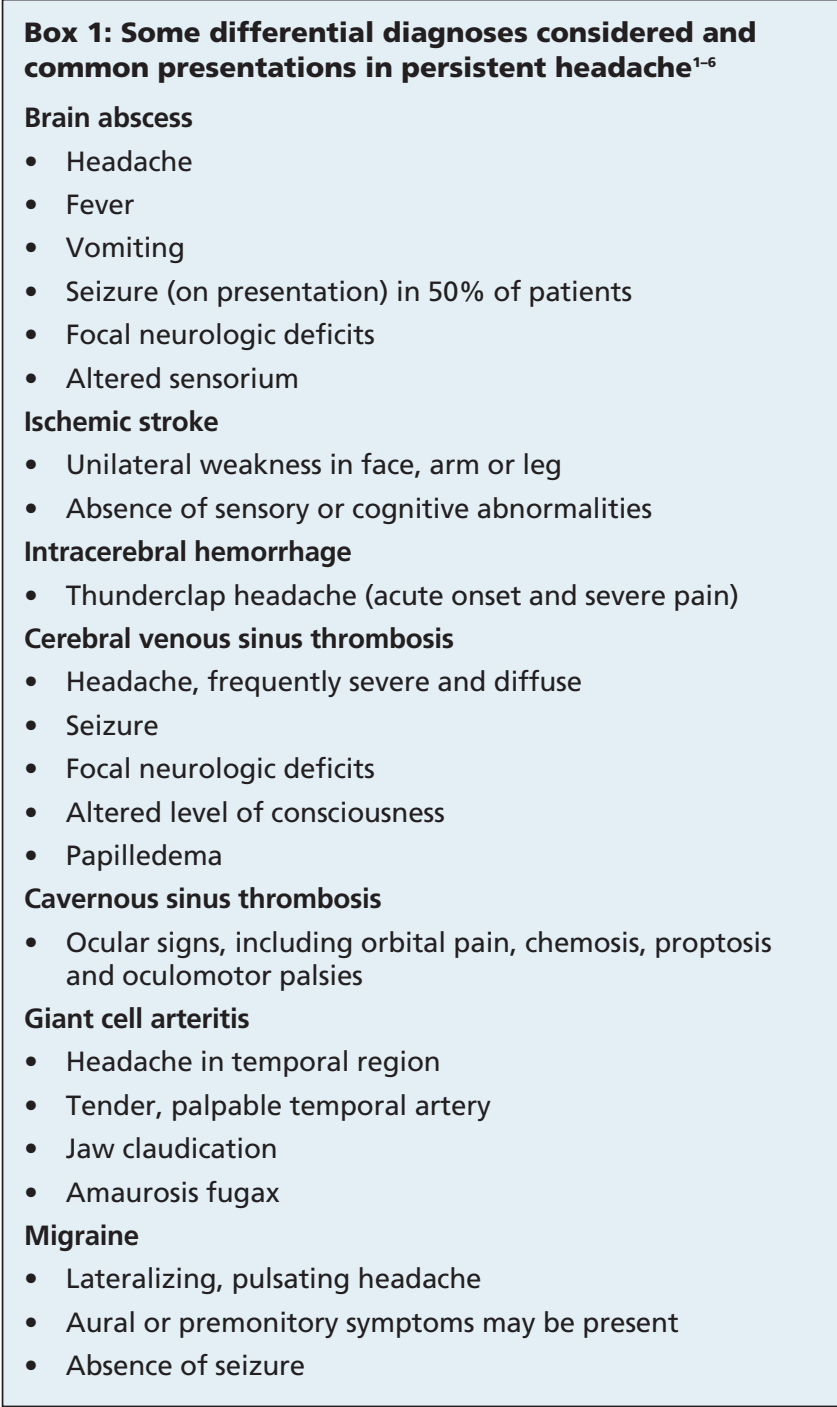

A cranial CT scan may be done if MRI is not readily available or if CT scanning is the type of imaging done initially in the emergency department, as is commonly the case. The cranial CT scan may reveal an acute cerebral disorder, venous infarcts or hemorrhages. The most frequently observed direct sign of cerebral venous sinus thrombosis, seen in $25 \%-30 \%$ of patients, is the "empty triangle" or delta sign, which is a non-filling of the confluens sinuum seen on CT images after injection of contrast medium (Figure 3). However, the CT scans may appear normal in $25 \%-30 \%$ of patients. ${ }^{1}$ The diagnostic value of CT scanning is greatly improved when coupled with CT venography, with a sensitivity of 75\%-100\% and a specificity of $81 \%-100 \% .{ }^{11}$ If the diagnosis is uncertain after MRI, a cerebral angiogram, previously considered the "gold standard," can be performed to provide better details of the cerebral veins.

Most patients with recent cerebral venous sinus thrombosis have elevated D-dimer concentrations. A negative D-dimer result is useful in ruling out the condition in patients who present with encephalic signs; however, a negative result cannot rule out the condition in patients with a recent isolated headache. ${ }^{9}$

\section{Management}

Current guidelines, supported by the American Academy of Neurology and the American College of Chest Physicians, recommend the use of low-molecular-weight heparin or weight-adjusted intravenous heparin as initial treatment even

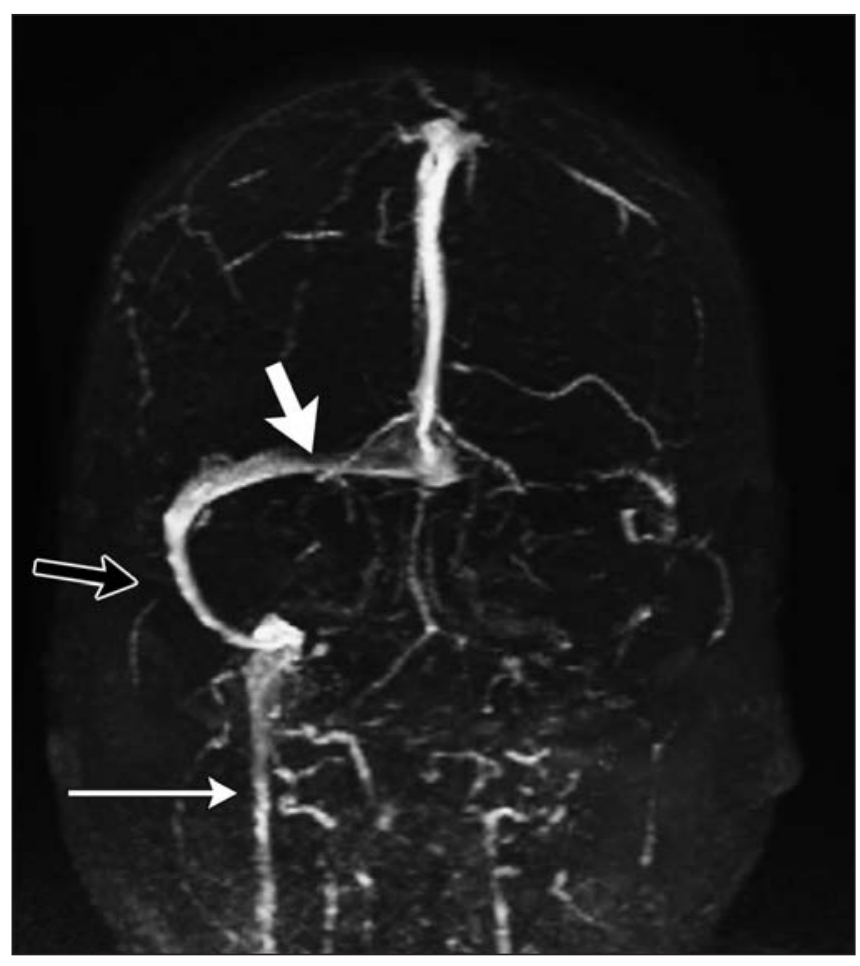

Figure 5: Magnetic resonance venogram showing normal right transverse sinus (thick white arrow), sigmoid sinus (black arrow) and internal jugular sinus (thin white arrow). The corresponding sinuses and internal jugular vein on the left side are not visible because of thrombosis. 
in the presence of hemorrhagic infarction, followed by oral anticoagulation with a vitamin $\mathrm{K}$ antagonist. ${ }^{12,13}$ Concomitant intracranial hemorrhage is not a contraindication to anticoagulation; however, risks and benefits should be weighed on an individual basis. The underlying cause of the thrombosis should be sought and treated.

The optimal duration of anticoagulation treatment after the acute phase is unknown because of the lack of randomized clinical trials. The European Federation of Neurological Societies 2006 guidelines suggest treatment with a vitamin K antagonist for three months in patients with a transient risk factor, six months in those with idiopathic cerebral venous sinus thrombosis or a weak hereditary thrombophilia, and possibly an indefinite duration in patients who present with a recurrent cerebral venous sinus thrombosis or have a severe hereditary thrombophilia. ${ }^{6}$

Prophylactic antiepileptic treatment is an option in patients presenting with a focal neurologic deficit and a focal parenchymal lesion on CT or MRI scans on admission. ${ }^{6}$

\section{Prognosis}

Anticoagulation results in good recovery with minor or no residual symptoms in $79 \%$ of patients. ${ }^{2}$ The presence of a neurologic deficit, cancer, altered mental status, involvement of the deep cerebral venous system, male sex, infection of the central nervous system, intracranial hemorrhage and coma are predictors of poor outcome. ${ }^{2}$ Coma on admission is the most consistent and strongest predictor of poor outcome. ${ }^{6}$ Isolated intracranial hypertension is not considered a predictor of poor outcome. ${ }^{2}$ Seizures do not have a significant independent influence on long-term outcome, but patients presenting with seizures are at increased risk of recurrent seizures within two weeks, especially those who have supratentorial lesions. ${ }^{10}$ Elderly patients are more likely to experience recurrent thrombotic events and incomplete recovery. ${ }^{7}$

This article has been peer reviewed.

Competing interests: None declared.

\section{REFERENCES}

1. Stam J. Thrombosis of the cerebral veins and sinuses. N Engl J Med 2005;352:1791-8.

2. Ferro JM, Canhao P, Stam J, et al.; ISCVT Investigators. Prognosis of cerebral vein and dural sinus thrombosis: results of the International Study on Cerebral Vein and Dural Sinus Thrombosis (ISCVT). Stroke 2004;35:664-70.

3. Honda H, Warren DK. Central nervous system infections: meningitis and brain abscess [review]. Infect Dis Clin North Am 2009;23:609-23.

4. Bernardini GL. Diagnosis and management of brain abscess and subdural empyema. Curr Neurol Neurosci Rep 2004;4:448-56.

5. Masuhr F, Mehraein S, Einhaupl K. Cerebral venous and sinus thrombosis. J Neurol 2004;251:11-23.

6. Einhaupl K, Bousser MG, de Bruijn SF, et al. EFNS guideline on the treatment of cerebral venous and sinus thrombosis. Eur J Neurol 2006;13:553-9.

7. Ferro JM, Canhao P, Bousser MG, et al.; ISCVT Investigators. Cerebral vein and dural sinus thrombosis in elderly patients. Stroke 2005;36:1927-32.

8. Ferro JM, Canhao P, Stam J, et al. Early seizure in cerebral vein and dural sinus thrombosis. Stroke 2008;39:1152-8.

9. Bousser MG, Ferro JM. Cerebral venous thrombosis: an update. Lancet Neurol 2007:6:162-70.

10. Idbaih A, Boukobza M, Crassard I, et al. MRI of clot in cerebral venous thrombosis. Stroke 2006;37:991-5.

11. Dormont D, Anxionnat R, Evrard S, et al. MRI in cerebral venous thrombosis. J Neuroradiol 1994;21:81-99.

12. Sacco RL, Adams R, Albers G, et al. Guidelines for prevention of stroke in patients with ischemic stroke or transient ischemic stroke. Circulation 2006;113:409-49.

13. Albers GW, Amarenco P, Easton JD, et al. Antithrombotic and thrombolytic therapy for ischemic stroke: American College of Chest Physicians evidence-based clinical practice guidelines (8th edition). Chest 2008;133:630S-669S.

Correspondence to: Dr. Anita Au, Department of Medicine, University of Alberta, Rm. 2F1, Walter C. Mackenzie Health Sciences Centre, 8440-112 St., Edmonton AB T6G 2R7; aau@ualberta.ca 